\title{
Correction to: The Minimum Sampling Rate and Sampling Duration When Applying Geolocation Data Technology to Human Activity Monitoring
}

Yan Zeng, Paolo Fraccaro, and Niels Peek

\begin{abstract}
Correction to:
Chapter "The Minimum Sampling Rate and Sampling

Duration When Applying Geolocation Data Technology

to Human Activity Monitoring" in: D. Riaño et al. (Eds.):

Artificial Intelligence in Medicine, LNAI 11526,

https://doi.org/10.1007/978-3-030-21642-9_29
\end{abstract}

Unfortunately the first author's name was spelled incorrectly. In the contribution it read

"Yan Zheng" but it should have read "Yan Zeng". 